\title{
Urgences
}

\section{Le littéral et le banal}

Denis Bellemare

Numéro 30, décembre 1990

L'autre du texte

URI : https://id.erudit.org/iderudit/025627ar

DOI : https://doi.org/10.7202/025627ar

Aller au sommaire du numéro

Éditeur(s)

Urgences

ISSN

0226-9554 (imprimé)

1927-3924 (numérique)

Découvrir la revue

Citer cet article

Bellemare, D. (1990). Le littéral et le banal. Urgences, (30), 70-78.

https://doi.org/10.7202/025627ar d'utilisation que vous pouvez consulter en ligne.

https://apropos.erudit.org/fr/usagers/politique-dutilisation/ 


\section{Le littéral et le banal}

\section{Denis Bellemarre}

Il paraîtra surprenant pour ceux qui connaissent le travail de Jacques Leduc de voir son dernier film, Trois pommes à côté du sommeil (1988), chapeauté de ce titre. Le réalisme extrême de ses œuvres précédentes - Tendresse ordinaire (1973), On est loin du soleil (1970) - répond mieux à première vue et à première lecture à cette appellation. Dans des articles antérieurs sur les films de Leduc ${ }^{1}, \mathrm{j}^{\prime}$ ai tenté de montrer que le désir d'emprise du réel sur le récit creuse, sous la lisse surface diégétique, des espaces en défaut. Le réel glisse vers d'autres objets et se dérobe comme perte mélancolique de cette réalité même. L'analyse saisit le film qui, à trop insister pour faire la preuve du réel, est pris en flagrant délit d'actions secrètes, insoupçonnées sous son impression de réalité. L'analyse prend le film à témoin de sa propre démarche langagière, tentant ainsi de le démarquer des habituels discours épuisés de québécitude. Et ce qui étonne dans ce réalisme, c'est non pas le manque d'imaginaire, mais plutôt l'imaginaire du manque, l'inscription rupturante d'un imaginaire en négatif. En ce sens, les films de Leduc remplissent bien ce programme.

Mais Trois pommes d côté du sommeil fait-il exception? Le rêve du début du film, la plasticité des images, les incessants allers et retours du présent au passé, l'évocation planétaire d'un sens du monde, la conception polyphonique des sons, des bruits, des musiques, tous ces éléments nous mettent en présence d'un signifiant en plein travail, à l'opposé d'un texte littéral qui, en quelque sorte, ferait le mort. L'accentuation de ces aspects formels appelle la différence. Mais cette accentuation, cette prolifération des points de vue, cette quantité d'effets questionnent, justement, au même titre que leur nivellement, leur rareté, leur absence. Car le littéral et le banal, pris dans leurs acceptions communes, à la lettre pour l'un, au familier pour l'autre, n'en révèlent pas

1 a La mélancolie et le banal ", Dérives, Montréal, $n^{\circ} 52\left(n^{\circ}\right.$ intitulé Nouveaux regands sur le cinéma québécois), septembre 1986; "Le demier objet ", Copio zéro, Montréal, $\mathrm{n}^{\circ} 30$ ( $\mathrm{n}^{\circ}$ intitulé Le documentaire vers de nouvelles voies), décembre 1986; "Les négativités ". Revue belge du cinéma. Bruxelles, n 27 ( $n^{\circ}$ intitulé Imaginaires du cinéma québécois, automne 1989. 
moins le travail fort particulier du signifiant à homogénéiser, à niveler, à abolir, à disparaître derrière le réel, à le faire avancer. Ces deux termes - le littéral et le banal - doivent être détachés de leurs connotations péjoratives et compris dans leur négativité constructive. Et c'est dans cette même avancée, cette même mise à l'épreuve du récit que Leduc, dans Trois pommes a côté du sommeil, ouvre les pans de résistance à la production textuelle, les innerve, tous ressorts des pare-excitations mis à découvert. L'imaginaire dans le cinéma québécois n'a jamais fait problème à mes yeux, tant il implique le réel comme une de ses modalités afin de lui restituer une partie auparavant supprimée. Mais la relation au symbolique s'avère d'un abord difficile. Et c'est selon ce parcours complexe que viennent s'inscrire le littéral et le banal dans Trois pommes à côté du sommeil.

Le littéral vise ici toute la mise en difficulté des procédés discursifs pour tenter de niveler, malgré leurs multiples occurrences, les différents points de vue. Il craint la menace de l'émergence du texte producteur et ne se sent à l'abri que dans l'énoncé monstrateur du figuré. La moindre agitation du signifiant l'atteint. Trois pommes rend bien cette secousse textuelle entre transparence et énonciation, manifestant ses couches de résistance, latentes et tues dans les autres films de Leduc. Mais ce film réussit-il bien à gérer les divers degrés du procès narratif sans dénier le signifiant cinématographique: lui, ce film, et Lui, le personnage principal de ce film, tous deux porteurs d'un imaginaire en négatif?

Le banal, à sa manière, relance le littéral. À l'activité interprétative, il supplée le rationnel, l'objectif, le vrai. Son projet ne se trame pas sur un fond imaginaire, il met toute son énergie à dire la primauté de la perception extérieure. Le banal met le triple accent sur la réalité, la perception et le familier, pour les arracher à leur lien avec l'imaginaire, la projection et l'étrange. Les faits réels, la perception engagent une certaine élaboration, voire une perlaboration, tout comme le littéral fraye les bords du texte. L'analyse se méfie des apparences, de la transparence, elle les prend en otage dans le jeu des surfaces représentationnelles. Ces deux concepts, le littéral et le banal, par une sorte de spécularisation symétrique, tentent de constituer une connaissance directe de l'objet sans intermédiaire symbolique. Nous tenterons d'éclairer leurs mises en œuvre dans Trois pommes à côté $d u$ sommeil. 
Le banal met en jeu une certaine conception du langage. II nous invite à méditer le fait que les deux póles du discours ne sont pas la métaphore et la métonymie, mais plus généralement le littéral et le figuré. ${ }^{2}$

Trois pommes d̀ côté du sommeil présente, dit la narratrice en voix off, "une journée dans la vie d'un homme d'aujourd'hui. Une journée qui se déroule au fil des événements et de la mémoire. " Lui, le personnage principal du film, n'a ni nom ni prénom, se lève en ce jour de ses quarante ans. Et au gré de ses nombreuses activités quotidiennes: sports, courses, travail de recherche à la pige, dîner, souper, il se remémore des moments particuliers de sa vie avec sa femme (dont il vient de se séparer), les nombreuses relations amoureuses toutes plus ou moins mal terminées et une amitié intellectuelle fondatrice avec l'astrophysicien Hubert Reeves. Ses travaux le poussent à réfléchir, dans une sorte d'appréhension du sens du monde, sur les équivalences toutes pascaliennes de l'infiniment grand, de l'infiniment petit de l'univers. Voué à une perception excessive de soi et du monde extérieur, il en cherche l'analyse, l'objectivation. Complètement désinvesti de tout affect amoureux - - Je n'ai pas cessé de t'aimer toi tout particulièrement, Madeleine. Jaai seulement cessé d'avoir de l'amour à donner, c'est tout $*$, dit-il à sa femme - il surinvestit l'ordre du réel. Le sens devient chose. Sujets, actes et affects se répercutent dans sa perception consciente pour se lire en gens, faits et gestes cristallisés dans leur réalité événementielle. * Le sujet devient objet et l'objet sujet cependant que s'accroît la dépossession de soi comme de toute chose. " 3

Or, dans cet infini du dehors, cet extérieur de soi, quelles formes d'expression et de contenu singularise ce texte filmique? En relation avec le symbolique, l'aspectualisation des nombreuses voix énonciatrices indique une piste qui a partie liée avec les formes et les stratégies de l'énonciation. Dans Linguistique et psychanalyse 4 , Michel Arrivé, marquant la relation qui s'institue entre la production symbolique et la procédure spécifique de l'énonciation qu'est l'embrayage, cite Greimas et Courtés :

2 Sami-Ali, Le banal, coll. "Connaissance de l'inconscient ", Paris, Gallimard, 1980, p. 13.

3 lbid., p. 37.

4 Michel Arrivé, Linguistique et psychanalyse, Paris, Méridien/Klincksieck, 1986, 180 p. 
L'embrayage produit une dé-référentialisation de l'énoncé qu'il affecte: ainsi la description de la nature se transforme en « état d'âme", l'enfance de Marcel [Proust], mémorisée [...]. cesse d'être une suite d'u événements" pour devenir une organisation $\propto$ figurative " de $\propto$ souvenirs $» .5$

Et pourtant ces aspects de la vie intérieure rappellent l'univers diégétique de notre personnage. Que se passe-t-il pour que ce qui peut faire figure reste au figuré? Quels mécanismes se mettent en place pour bloquer ou tout au moins faire obstacle à la voie symbolisante? Nous retiendrons ces trois termes utilisés dans la précédente citation - référentialisation, description, suite d'événements -, ils vont relancer de façon dynamique le littéral et le banal.

Dans la pluralité des scènes rompues par la circulation des souvenirs de notre personnage, la relation entre la production symbolique et l'énonciation s'institue avec équivoque. Les allers et retours du passé au présent ne produisent pas l'effet d'identification attendu entre sujet d'énoncé et sujet d'énonciation. L'impossibilité de maintenir une réelle différence dans les termes opposés fait ressortir des modalités particulières de l'énonciation et de la mémoire. Ce personnage extérieur à lui-même se fait redoubler par une instance narrative objectivante. Lui est un non-je. La voix off de la narratrice l'instaure dans la catégorie d'homme d'aujourd'hui, le singulier appelle le général, le sujet devient objet d'observation, la vie se fait description d'événements. * Les événements de la vie de cet homme, dit la narratrice, ne se disposent pas de façon chronologique. Dans une vie, chaque événement nouveau vient toujours influencer le sens des autres. * Cette tendance à la systématisation se retrouve dans deux syntagmes en accolade ${ }^{6}$ importants du film. Cette segmentation cherche à regrouper un ensemble de plans a-chronologiques sous une même parenté d'idée, une même catégorie de faits. Madeleine apprend qu'elle est enceinte et se demande bien qui l'élèvera, cet enfant-là: six plans de différents personnages de milieux sociaux divers se succèdent. Lui se promène en voiture et exprime le besoin d'une rencontre sexuelle: quatorze plans de couples se suivent qui

5 A. J. Greimas et J. Courtes, Sémiotique: dictionnaire raisonné de la théorie du langage, Paris, Hachette Université, p. 121.

6 Christian Metz, * Problèmes de dénotation dans le film de fiction ", Essais sur la signification au cinóma, tome 1, Paris, Klincksieck, 1971, p. 127. 
traversent la rue, se promènent, retournent à la maison. Dans le premier cas, la question de la responsabilité personnalisée se déplace vers une représentation sociale. Dans le deuxième cas, tous les couples composent la notion de couple. Ce type d'enchaînement nie la singularité du plan au profit d'une généralité. "Le banal n'est rien d'autre que le singulier en général. " 7

Lui revendique d'une certaine manière la place de l'observateur. Comme personnage, il oscille entre son rôle d'informateur et d'observateur, comme sujet d'énoncé et sujet d'énonciation, il entremêle les fonctions d'embrayage et de débrayage. Les structures d'énonciation rejoignent diégétiquement les plaintes constantes de sa femme, de ses maîtresses, de ses amis, sur ses difficultés d'implication, d'intériorisation, voire de silence intérieur. L'instance narrative supérieure énonce ses pensées comme des faits. Nous nous retrouvons face à un sujet hyper-cognitif. "J'analyse, dit-il, pour mieux comprendre et à force d'analyser, la matière que j'analyse finit par perdre son sens. * Le haut degré de référentialisation conduit à la question du rôle imparti à la mémoire du personnage, à la mémoire du film où la mise en discours de cette vie intérieure tend vers un réalisme objectif.

Le problème ne se situe pas au niveau diégétique pour le spectateur qui perçoit bien les démarcations temporelles, mais peut-être un peu moins les différenciations tant la relation du présent au passé s'opère dans une sorte d'interchangeabilité des mots, des gestes, des gens. La quantité des points de vue se fait toujours sous le même éclairage, dans la même clarté de l'objet référent. Dans cette image objective et concrète, la mémoire ne semble garder aucune trace du passage des affects. L'image du rappel au passé demeure intacte: elle ne soulève que de rares pans du signifiant mnésique, n'en retenant que du signifié, de l'informatif, comme si la symbolisation du manque s'avérait irréalisable. La mémoire, opératoire, historisante, se remémore de l"actuel, du factuel, et dans cette faille de la représentation, aucune zone d'ombre ne vient restaurer la perte d'investissement.

La relation au référent ne représente pas un chaînon, mais une rupture, un non-sens a-signifiant $[\ldots]$, cette relation 
correspond à une présentation sous la forme d'une perception concrète. 8

L'objet référent, la présentation s'ajoutent à la perception et à la référentialisation pour compléter le tableau de la difficulté de la re-présentation. Le littéral et le banal, dans leur symétrique spécularité, cherchent à reproduire du même, du général, à homogénéiser les différents écarts textuels. Les formes l'emportent sur l'affect, la systématisation sur l'intuition, l'objectivité sur la subjectivité.

Cette symétrie se retrouve le long du film comme une lecture dirigée du parcours de la mémoire. Elle empêche de faire figure tant elle est rivée, travaillée par des structures de répétition, d'équivalence, de complétion. Le montage, nous le verrons bientôt, tente d'arrimer sans heurt, avec logique et acharnement, ces bribes d'un moi morcelé dans un système où la rationalité domine à vouloir être une, univoque, uniformatrice du et dans le réel. Toutes ces voies conduisent à une impasse symbolisante de la représentation. Par perlaboration du travail filmique, nous désignons cette reprise interminable à ne pouvoir nommer et non dire, à figurer et non montrer, à représenter et non présenter, à symbolịser et non réaliser le véritable objet de sa perte. «J'ai seulement cessé d'avoir de l'amour à donner, c'est tout „, cette phrase de Lui revient par trois fois dans le film. Nicos Nicolaïdis distingue la notion de perte des notions de manque et d'absence en ce que celles-ci sont liées à la condition métaphorisante et symbolisante de la représentation,tandis que la perte s'applique aux mécanismes imageants. Il nous définit clairement l'issue à cette a-symbolisation.

C'est par l'asymétrie, que nous avons aussi appelé réfraction dioptique, que le sujet peut sortir de cette captation en miroir, dans la mesure où le sujet atteint ce maximum asymétrique caractéristique du langage qui, cassant la compulsion de répétition, par l'irréductibilité polysémique du signifiant rend toute substitution possible i.e. toute métaphore possible. ${ }^{9}$

Le montage s'avère la forme d'expression cinématographique toute désignée pour la juxtaposition d'éléments hétérogènes faisant surgir de deux ou plusieurs plans une

8 Nicos Nicolaïdis, La représentation. Essai psychanalytique, coll. «Psychismes ", Paris, Dunod, 1984, p. 48.

9 Ibid., p. 15. 
76

configuration inattendue. Trois pommes à côté du sommeil comme Hiroshima mon amour d'Alain Resnais sont tous deux des films de montage qui explorent l'architecture de la mémoire. Leur opposition ne se joue pas sur leur valeur réciproque, mais leur comparaison permet de distendre deux pôles, deux points de repère dans l'infini des figures. Hiroshima joue du contrepoint imagique et sonore. Le passé et le présent se changent mutuellement dans une lecture avivée de leurs sensations, et le présent en ressort différé. Par l'instabilité et la contamination des deux registres du réel et de l'imaginaire, la blessure amoureuse vient se symboliser sur fond de Deuxième Guerre mondiale. Se vit alors une traversée des apparences dans un monde où les affects sont fortement investis. Trois pommes relie le présent et le passé de façon plus frontale. Leurs liens mémoriels fortement stabilisés se basent sur la ressemblance, l'identité, et n'altèrent en aucune manière leur rapport, leur perception. La traversée ne se fait plus dans les deux sens. Le présent fait intervenir le passé comme garant de l'actuel. La mémoire ne retient le passé qu'à la surface de la pensée actualisée, s'approchant ainsi de la pensée opératoire définie par Marty cité par Sami-Ali: «La "pensée opératoire" [...] se ramène à détailler un acte ou une succession d'actes précis toujours intimement liés à une réalité actuelle. " 10

Le montage s'assemble autour de thèmes et de tons comme autant d'activités de connaissance afin de mieux saisir toutes les données objectives du présent et du passé rétablissant la matérialité d'un fait. Le montage, à l'instar du littéral et du banal, se structure autour de trois procédés: la répétition, l'interchangeabilité, la complémentarité. La répitition dédouble les contenus et rend invisible le moindre déplacement. Cette façon donne l'impression d'accumulation quantitative et n'offre aucune prise à un possible changement qualitatif de la figure. D'une image l'autre, aucun glissement paradigmatique ne s'effectue. Des phrases, des mouvements, des gestes servent de relais. Madeleine et Lui dînent au restaurant pour fêter son anniversaire. La conversation rappelle vite la redondance de certaines situations. Lui dit un mot au restaurant: «J'ai rien à dire ». Et ce mot d'aujourd'hui nous apporte la réponse de Madeleine l'automne passé: «Dis pas que tu n'as rien à dire. Dis plutôt... "Cynique, connais- 
sant le prix de tout et la valeur de rien, il lui rétorque: "Quand on parle, c'est qu'on a rien à dire. Quand on parle pas, ça veut tout dire. Toujours au restaurant, Lui s'entend dire: «M'écoutes-tu quand je te parle? » et il se remémore la même question criée au pied d'un escalier, un été précédent. Dans cette captation en miroir, les plans réactivent la même scène non résolue, toujours ouverte à la compulsion de répétition.

La familiarité de l'objet et son caractère répétitif ne suffisent pas à définir le banal en son essence propre... Le banal a partie liée avec l'épuisement du contenu émotionnel et cognitif de l'objet, moyennant une répétition qui ne manque pas d'engendrer un équivoque sentiment de monotonie. ${ }^{11}$

Les plans se répondent l'un l'autre en écho. Le tissu filmique, perméable à toute intervention sonore, nivelle les différences. Une continuité œuvre, via la complétion du passé par le présent, du présent par le passé, à court-circuiter les points de vue. Le désir de symétrie transige au delà de toutes les temporalités. Madeleine et Lui font l'amour; le deuxième plan suivant, Lui, tout essoufflé de son exercice de natation dans une piscine, repose satisfait, sur le dos. Hubert Reeves se ballade avec Lui à la campagne et parle de son manque de silence intérieur; le plan suivant, Pascale, une des maîtresses de Lui, debout à la terrasse d'un immeuble en ville, exprime son amour du bruit qui empêche parfois de penser. Madeleine, dans son jardin, se pose la question d'une vie sans responsabilité; le plan suivant, toujours à la campagne avec Lui, Hubert Reeves réplique: «Sans responsabilité, ça dépend de quelle responsabilité on parle." Ce processus de complémentarité souligne la mesure de Lui comme observateur de toute chose. Il collectionne les multiples points de vue pour les assujettir à son regard analytique dominant. Un autre mécanisme vient parfaire la programmation de cette pensée opératoire et compléter la géométrie du banal dans un système des équivalences et des interchangeabilités. L'infiniment grand et l'infiniment petit se rejoignent. Lui, chroniqueur scientifique à la pige, examine sous la loupe la géographie de sa main, de sa peau; suivent plusieurs plans de paysages ainsi modulés. Il existe une poésie et une esthétique du banal; c'est le moment plastique du film, mimétique du chant du monde

11 Ibid., p. 23. 
comme Roger Caillois le réfléchit, où toutes les formes appellent la forme. Chez le personnage principal, cette obsession de système et de circularité chemine de la fourmi à la pomme, de la lune à la terre, de la planète à la galaxie dans une sorte de rebondissement nucléé, organique, où le moi reste en plan, consommé. Où de la brique au cœur, le lapsus de notre chroniqueur scientifique sur l'oxydation des sentiments (sédiments) se dit et ment à Pascale, l'amoureuse.

L'objet est une surface reliant d'autres surfaces qui sont indifféremment des objets ou ce qui sépare les objets. Figure et fond sont interchangeables, de même que vide et plein s'équivalent rigoureusement. ${ }^{12}$

$$
* * *
$$

Cet article sur le littéral et le banal s'inscrit dans une recherche plus vaste sur la mélancolie dans le cinéma québécois. Cet essai cherche à cerner des formes d'expression et de contenu originales, spécifiques. La mélancolie, le banal et le littéral appartiennent au vocabulaire commun. La très grande tâche consiste à affiner, à affûter ces concepts théoriquement et méthodologiquement pour l'analyse textuelle d'un corpus particulier, de films singuliers. Le littéral et le banal posent la question de la figurabilité dans le texte filmique, de son inscription; en ce sens, ils redoublent la difficulté de la représentation de la perte mélancolique profondément ancrée dans le cinéma québécois, notamment dans les films de Jacques Leduc. Il ne s'agit pas d'enfermer ces films dans un diagnostic clos, mais bien de faire travailler leurs résistances. Les formes du littéral et du banal s'ajoutent de façon dynamique aux problèmes d'introjection dans le deuil et la mélancolie.

Dans la magie incorporante, on relève ainsi deux procédés conjugués: la démétaphorisation (la prise au pied de la lettre de ce qui s'entend au figuré) et l'objectivation (ce qui est subi n'est pas une blessure du sujet mais la perte d'un objet). ${ }^{13}$

12 Ibid., p. 51.

13 Nicolas Abraham et Maria Torok, L'écorce et le noyau, Paris, Aubier Montaigne, 1978, p. 261. 